\title{
PROBABILITY MEASURE FUNCTORS PRESERVING THE ANR-PROPERTY OF METRIC SPACES
}

\author{
NGUYEN TO NHU AND TA KHAC CU
}

(Communicated by James West)

\begin{abstract}
Let $P_{k}(X)$ denote the set of all probability measures on a metric space $X$ whose supports consist of no more than $k$ points, equipped with the Fedorchuk topology. We prove that if $X \in$ ANR then $P_{k}(X) \in$ ANR for every $k \in \mathbf{N}$. This implies that for each $k \in \mathbf{N}$ the functor $P_{k}$ preserves the topology of separable Hilbert space.
\end{abstract}

\section{INTRODUCTION}

For a metric space $X$ let $F(X)$ denote the linear space of all functions of finite support on $X$ equipped with the $C_{b}(X)$-topology, that is, the weak topology induced by the set $C_{b}(X)$ of all bounded continuous functions on $X$. Observe that the space $F(X)$ with the $C_{b}(X)$-topology is a locally convex space. Every function $\mu \in F(X)$ can be written in the form $\mu=\sum_{i=1}^{k} m_{i} \delta x_{i}$, where $m_{i} \in \mathbf{R}$ for $i=1, \ldots, k$ and $\delta_{x}$ denotes the Dirac function with support at $x$, that is

$$
\delta_{x}(y)= \begin{cases}1 & \text { if } x=y \\ 0 & \text { if } x \neq y\end{cases}
$$

We denote

$$
\|\mu\|=\sum_{i=1}^{k}\left|m_{i}\right| \text { and } \operatorname{supp} \mu=\left\{x_{1}, \ldots, x_{k}\right\} .
$$

Following Fedorchuk [Fe] let us say that a function $\mu \in F(X)$ is a probability measure iff $\mu(x) \geq 0$ for each $x \in X$ and $\|\mu\|=1 . \mu(x)$ is called the mass of $\mu$ at $x$.

For each $k \in \mathbf{N}$ let $P_{k}(X)$ denote the set of all probability measures on $X$ whose supports consist of no more than $k$ points and let $P_{\infty}(X)=\bigcup_{k=1}^{\infty} P_{k}(X)$.

Received by the editors June 7, 1988. Presented to Spring Topological Conference, Gainsville, Florida, April 7, 1988.

1980 Mathematics Subject Classification (1985 Revision). Primary 60B05, 54C55, 58D15, 58B05; Secondary 57N20, 54C35.

Key words and phrases. ANR, probability measure, Hilbert space.

This paper was written while the first author was visiting Texas Tech University. The first author would like to express his thanks to Texas Tech University for their hospitality and financial support. 
We shall see in $\S 2$ that the $C_{b}(X)$-topology and the topology introduced by Fedorchuk [Fe] are equivalent on $P_{\infty}(X)$.

Let us observe that $P_{\infty}(X)$ is a convex set in the locally convex space $F(X)$. Therefore $P_{\infty}(X)$ is an absolute extensor for any metric space $X$, hence $P_{\infty}(X) \in \mathrm{AR}$ iff it is metrizable.

In [Fe] Fedorchuk proved that if $X$ is a compact ANR-space then $P_{k}(X) \in$ ANR for every $k \in N$. The aim of this note is to prove Fedorchuks' theorem without the compactness assumption on $X$. The compactness of $X$ is essential in the proof of Fedorchuk [Fe]. In our case we use a different approach which involves a characterization of ANR-spaces established by the first author in $[N 1]$. Our proof uses an idea of $[N 1]$, see also $[N 1, N 2]$, however in our case masses of probability measures lead to more complicated situations.

The proof of the main result of this note is given in $\S 3$. In $\S 2$ we describe the Fedorchuk topology for the space $P_{\infty}(X)$ and show that $P_{k}(X)$ is metrizable for any $k \in \mathbf{N}$. This is the first step toward our result.

\section{FedorchuK's topology on $P_{\infty}(X)$ AND the Metrizability of $P_{k}(X)$}

In this section we describe Fedorchuk's topology on $P_{k}(X)$ and prove that $P_{k}(X)$ is metrizable for any $k \in \mathbf{N}$. For each $\mu_{0}=\sum_{i=1}^{k} m_{i}^{0} \delta x_{i}^{0} \in P_{\infty}(X)$ we define a neighborhood basis of $\mu_{0}$ of the form $\mathscr{O}\left\langle\mu_{0}, U_{1}, \ldots, U_{k}, \varepsilon\right\rangle$ where $\varepsilon>0$ and $U_{1}, \ldots, U_{k}$ are disjoint neighborhoods of $x_{1}^{0}, \ldots, x_{k}^{0}$, respectively.

$$
\begin{aligned}
\mathscr{O}\left\langle\mu_{0},\right. & \left.U_{1}, \ldots, U_{k}, \varepsilon\right\rangle \\
= & \left\{\mu \in P_{\infty}(X): \mu=\sum_{i=1}^{k+1} \mu_{i}, \operatorname{supp} \mu_{i} \subset U_{i}\right. \text { and } \\
& \left.\left|m_{i}^{0}-\left\|\mu_{i}\right\|\right|<\varepsilon, i=1, \ldots, k \text { and }\left\|\mu_{k+1}\right\|<\varepsilon\right\} .
\end{aligned}
$$

Observe that $U_{i}, i=1, \ldots, k$ can be taken from a fixed basis for $X$. The following fact shows that $P_{\infty}(X)$ is an absolute extensor for any metric space $X$.

(2.1) Proposition. The Fedorchuk topology and the $C_{b}(X)$-topology are equivalent on $P_{\infty}(X)$.

Proof. For $\mu=\sum_{i=1}^{k} m_{i} \delta x_{i} \in P_{\infty}(X)$ and $f \in C_{b}(X)$ we denote $\sum_{i=1}^{k} m_{i} f\left(x_{i}\right)$ by $\int f d \mu$.

At first we assume that $\mu_{n} \rightarrow \mu=\sum_{i=1}^{k} m_{i} \delta x_{i}$ in the Fedorchuk topology. Let $\varepsilon>0$ and $f \in C_{b}(X)$ be a bounded continuous function. For each $i=1, \ldots, k$ we take a neighborhood $U_{i}$ of $x_{i}$ such that $\left|f(x)-f\left(x_{i}\right)\right|<(1 / 3 k) \varepsilon$ for every $x \in U_{i}, i=1, \ldots, k$. Since $\mu_{n} \rightarrow \mu$ in Fedorchuk's topology there is an $n_{0} \in \mathbf{N}$ such that for every $n \geq n_{0}$ we have $\left|m_{i}-\left\|\mu_{n}^{i}\right\|\right|<\varepsilon / 3 M k$ for $i=1, \ldots, k$ and $\left\|\mu_{n}^{k+1}\right\|<\frac{1}{3} \varepsilon$, where $M=\sup \{\mid f(x): x \in X\}, U_{k+1}=$ $X \backslash \bigcup_{i=1}^{k} U_{i}$ and $\mu_{n}^{i}=\mu_{n} \mid U_{i}, i=1, \ldots, k+1$. Then for every $n \geq n_{0}$ we obtain $\left|\int f d \mu_{n}-\int f d \mu\right| \leq \varepsilon$. Therefore $\mu_{n} \rightarrow \mu$ in the $C_{b}(X)$-topology. 
Conversely, assume that $\mu_{n} \rightarrow \mu=\sum_{i=1}^{k} m_{i} \delta x_{i}$ in the $C_{b}(X)$-topology. Let $\mathcal{O}\left\langle\mu, U_{1}, \ldots, U_{k}, \varepsilon\right\rangle$ be a neighborhood of $\mu$ in the Fedorchuk topology. Take a Urysohn function $f_{0}: X \rightarrow[0,1]$ such that $f_{0} \mid X \backslash \bigcup_{i=1}^{k} U_{i}=1$ and $f_{0}\left(x_{i}\right)=0$ for $i=1, \ldots, k$. Since $\int f_{0} d \mu_{n} \rightarrow \int f_{0} d \mu$ we infer that there is an $n_{0} \in N$ such that setting $\mu_{n}^{i}=\mu_{n} \mid U_{i}, i=1, \ldots, k$ and $\mu_{n}^{k+1}=\mu_{n} \mid X \backslash \bigcup_{i=1}^{k} U_{i}$ we obtain

$$
\left\|\mu_{n}^{k+1}\right\|<\frac{1}{2} \varepsilon \text { for every } n \geq n_{0} .
$$

For each $i=1, \ldots, k$ let $f_{i}: X \rightarrow[0,1]$ be a Urysohn function such that $f_{i} \mid U_{i}=1$ and $f_{i} \mid U_{j}=0$ for $j \neq i$. Since $\int f_{i} d \mu_{n} \rightarrow \int f_{i} d \mu$ we infer that there is an $n_{i} \in \mathbf{N}$ such that for every $n \geq n_{i}$ we have

$$
\left|\left\|\mu_{n}^{i}\right\|+\int_{X \backslash \cup_{i=1}^{k} U_{i}} f_{i} d \mu-m_{i}\right|<\frac{1}{2} \varepsilon \quad \text { for } i=1, \ldots, k .
$$

Since

$$
\int_{X \backslash \cup_{i=1}^{k} U_{i}} f_{i} d \mu \leq\left\|\mu_{n}^{k+1}\right\| \quad \text { for } i=1, \ldots, k
$$

from (1) we get ||$\left|\mu_{n}^{i} \|-m_{i}\right|<\frac{1}{2} \varepsilon+\frac{1}{2} \varepsilon=\varepsilon$ for $i=1, \ldots, k$. Consequently, taking $\bar{n}=\max \left\{m_{i}, i=0, \ldots, k\right\}$ we obtain $\mu_{n} \in \mathscr{O}\left\langle\mu, U_{1}, \ldots, U_{k}, \varepsilon\right\rangle$ for every $n \geq \bar{n}$.

The proposition is proved.

Now we shall prove the main result of this section.

(2.2) Theorem. $P_{k}(X)$ is metrizable for any $k \in \mathbf{N}$.

The proof of Theorem 2.2 is based on the following fact due to Frink [Fr], see also [M].

(2.3) Theorem [Fr]. $A T_{1}$-space $X$ is metrizable if and only if the following condition holds:

(Fr) For each $x \in X$ there exists a neighborhood basis $\left\{U_{n}(x)\right\}_{n=1}^{\infty}$ such that if $U_{n}(x)$ is given there exists an $m=m(x, n)$ such that $U_{m}(y) \cap U_{m}(x) \neq \varnothing$ implies $U_{m}(y) \subset U_{n}(X)$.

Proof of Theorem 2.2. Obviously $P_{k}(X)$ is a $T_{1}$-space. Thus by Theorem 2.3 it suffices to verify the condition ( $\mathrm{Fr}$ ).

For each $\mu=\sum_{i=1}^{q} m_{i} \delta x_{i} \in P_{k}(X), q \leq k$ we define a neighborhood basis $\left\{\mathscr{O}_{n}(\mu)\right\}_{n=1}^{\infty}$ satisfying the condition (Fr). For each $i=1, \ldots, q$ we take $\left\{U^{n}\left(x_{i}\right)\right\}_{n=1}^{\infty}$ such that

$$
\begin{gathered}
\operatorname{diam} U^{n}\left(x_{i}\right)<\frac{1}{4} \min \left\{2^{-n}, \operatorname{dist}\left(U^{n}\left(x_{i}\right), U^{n}\left(x_{j}\right)\right) i \neq j\right\} \\
\left\{U^{n}\left(x_{i}\right)\right\} \text { satisfies the condition }(\mathrm{Fr}) .
\end{gathered}
$$

We put

$$
\mathscr{O}_{n}(\mu)=\mathscr{O}\left\langle\mu, U_{1}^{n}, \ldots, U_{k}^{n}, \varepsilon_{n}(\mu)\right\rangle
$$


where $U_{i}^{n}=U^{n}\left(x_{i}\right), i=1, \ldots, q$ and $\varepsilon_{n}(\mu)<\min \left\{2^{-n}, m_{i}, i=1, \ldots, q\right\}$. Let us show that $\left\{\mathscr{O}_{n}(\mu)\right\}_{n=1}^{\infty}$ satisfies (Fr).

Given $\mathscr{O}_{n}(\mu)$. Since $\varepsilon_{n}(\gamma)<2^{-n}$ for every $\gamma \in P_{k}(X)$ there exists an $m \in \mathbf{N}$ such that

$$
\varepsilon_{m}(\gamma)<\frac{1}{4 k} \min \left\{\varepsilon_{n}(\mu), m_{i} i=1, \ldots, q\right\} \text { for every } \gamma \in P_{k}(X) .
$$

We shall prove that $m(\mu, n)=\max \left\{m, m\left(x_{i}, n\right), i=1, \ldots, q\right\}$ satisfies the desired property of (Fr).

Assume that $\mathscr{O}_{n}(\gamma)=\mathscr{O}_{m}\left\langle\gamma, V_{1}^{m}, \ldots, V_{r}^{m}, \varepsilon_{m}(\gamma)\right\rangle$ with $\mathscr{O}_{m}(\gamma) \cap \mathscr{O}_{m}(\mu) \neq \varnothing$. Take $\theta \in \mathscr{O}_{m}(\gamma) \cap \mathscr{O}_{m}(\mu)$ and write $\theta_{i}=\theta \mid U_{i}^{m}, i=1, \ldots, q$ and $\theta_{q+1}=$ $\left.\theta\right|_{X \backslash \cup_{i=1}^{\varphi} U_{i}^{m}}, A_{i}=\operatorname{supp} \theta_{i}, \quad i=1, \ldots, q+1$. Since $\left\|\theta_{i}\right\| \geq m_{1}-\varepsilon_{m}(\mu)>$ $m_{i}-\frac{1}{4} m_{i}=\frac{3}{4} m_{i}>\varepsilon_{m}(\gamma), i=1, \ldots, q$ we infer that for every $i \leq q$ there exists at least $j \in\{1, \ldots, r\}$ such that $A_{i} \cap V_{j} \neq \varnothing$. Let

$$
G_{i}=\cup\left\{V_{j}: V_{j} \cap A_{i} \neq \varnothing\right\}, i=1, \ldots, q ; G_{q+1}=\cup\left\{V_{j}: V_{j} \subset X \backslash \bigcup_{i=1}^{q} A_{i}\right\} .
$$

Since $A_{i} \subset U_{i}^{m}$ from (3) it follows that

$$
G_{i} \subset U_{i}^{n} \text { for every } i=1, \ldots, q \text {. }
$$

We shall show that $\mathscr{O}_{m}(\gamma) \subset \mathscr{O}_{n}(\mu)$. For every $\omega \in \mathscr{O}_{m}(\gamma)$ we denote $\omega_{i}=$ $\omega \mid G_{i}$ for $i=1, \ldots, q+1 ; \omega_{i, j}=\omega_{i} \mid V_{j}$ for $V_{j} \subset G_{i} ; \theta_{i, j}=\theta_{i} \mid V_{j}$ for $V_{j} \subset G_{i}$. Since $\omega, \theta \in \mathscr{O}_{m}(\gamma)$ it follows that $\left\|\omega_{i, j}\right\|-\left\|\theta_{i, j}\right\| \mid<2 \varepsilon_{m}(\gamma)$. Note that $\operatorname{Card}\left\{j: V_{j} \subset G_{i}\right\} \leq r \leq k$. From (4) we obtain

$$
\|\| \omega_{i}\|-\| \theta_{i}\left\|\left|\leq \sum_{V_{j} \subset G_{i}}\right|\right\| \omega_{i, j}\|-\| \theta_{i, j} \| \mid<2 k \varepsilon_{m}(\gamma)<\frac{1}{2} \varepsilon_{n}(\mu)
$$

for every $i=1, \ldots, q+1$. Hence

$$
\left|\left\|\omega_{i}\right\|-m_{i}\right| \leq\left|\left\|\omega_{i}\right\|-\left\|\theta_{i}\right\|\right|+\left\|\theta_{i}-m_{i}\right\|<\frac{1}{2} \varepsilon_{n}(\mu)+\varepsilon_{m}(\mu)<\varepsilon_{n}(\mu)
$$

for every $i=1, \ldots, q$ and by (6) we have

$$
\left\|\omega_{q+1}\right\| \leq \theta_{q+1}+\frac{1}{2} \varepsilon_{n}(\mu) \leq \varepsilon_{m}(\mu)+\frac{1}{2} \varepsilon_{n}(\mu)<\varepsilon_{n}(\mu) .
$$

Consequently from (5) we infer that $\omega \in \mathscr{O}_{n}(\mu)$.

This completes the proof of Theorem 2.2.

\section{THE RESULTS}

Our result in this note is the following

(3.1) Theorem. If $X \in \mathrm{ANR}$ then $P_{k}(X) \in \mathrm{ANR}$ for each $k \in \mathbf{N}$.

As a consequence of Theorem 2.1 we get 
(3.2) Corollary. $P_{k}\left(\ell_{2}\right) \cong \ell_{2}$ for each $k \in \mathbf{N}$.

Here $\ell_{2}$ denotes separable Hilbert space, and $X \cong Y$ means $X$ is homeomorphic to $Y$.

Proof. Since $P_{k}\left(l_{2}\right) \in \mathrm{AR}$ the assertion follows from a result of [DT].

Let $\left\{\mathscr{U}_{n}\right\}$ be a sequence of open covers of a metric space $X$ and let $\mathscr{U}=$ $\bigcup_{n \in \mathbf{N}} \mathscr{U}_{n}$. By $\mathscr{N}(\mathscr{U})$ we denote the nerve of $\mathscr{U}$. We write $K \prec\left\{\mathscr{U}_{n}\right\}$ iff $K$ is a subcomplex of $\mathscr{N}(\mathscr{U})$ and for each simplex $\sigma \in K$ we have $\sigma \subset \mathscr{U}_{n} \cup \mathscr{U}_{n+1}$ for some $n \in \mathbf{N}$. We write

$$
N(\sigma)=\max \left\{n \in N: \sigma \subset \mathscr{U}_{n} \cup \mathscr{U}_{n+1}\right\} .
$$

The proof of Theorem 3.1 uses the following fact which is a slight modification of a charcterization of ANR-spaces given in [N1].

(3.3) Theorem [N1]. A metric space $X \in \mathrm{ANR}$ if and only if there exists a sequence of open covers $\left\{\mathscr{U}_{n}\right\}$ of $X$ such that for any $K \prec\left\{\mathscr{U}_{n}\right\}$ and for any selection $f: K^{0} \rightarrow X$ (i.e., $f(U) \in U$ ) there is a map $g: K \rightarrow X$ such that if $\left\{\sigma_{n}\right\}$ is a sequence of simplices of $K$ for which $f\left(\sigma_{n}^{0}\right) \rightarrow x_{0} \in X$ as $N\left(\sigma_{n}\right) \rightarrow \infty$ then we have $g\left(\sigma_{n}\right) \rightarrow x_{0}$.

Here we say that a sequence $\left\{A_{n}\right\}$ of subsets of a metric space $X$ tends to a point $x_{0} \in X$ iff $\operatorname{diam}\left(A_{n} \cup\left\{x_{0}\right\}\right) \rightarrow 0$.

Note that the proof of the characterization theorem of ANR-spaces given in [N1] proves also Theorem 3.3.

Proof of Theorem 3.1. The remaining part of this section is devoted to the proof of our main result. We shall verify the conditions of Theorem 3.3 for $P_{k}(X)$. Assume that $X$ is an ANR. Since every metric space can be embedded isometrically as a closed subset of a normed space, see [BP], by the ANR-property of $X$ without loss of generality we may assume that $X$ is an open subset of a normed space.

For each $n \in \mathbf{N}$ we take an open cover $\mathscr{W}_{n}$ of $X$ such that $\mathscr{W}_{n+1} \prec \mathscr{W}_{n}$ and $\operatorname{diam} W<2^{-n}$ for each $W \in \mathscr{W}_{n}$. Put $\mathscr{W}=\bigcup_{n=1}^{\infty} \mathscr{W}_{n}$. We shall assume that the Fedorchuk topology of $P_{k}(X)$ is induced by $\mathscr{W}$.

For each $n \in \mathbf{N}$ we take a cover $\mathscr{V}_{n}$ of $X$ consisting of open convex sets such that

$$
\begin{array}{cc}
\text { conv } V \prec \mathscr{W}_{n} & \text { for each } V \in \mathrm{St} \mathscr{V}_{n} \\
\mathscr{V}_{n+1} \prec \mathscr{V}_{n} & \text { for each } n \in \mathbf{N} .
\end{array}
$$


Set

$$
\tilde{\mathscr{U}}_{n}=\left\{\mathscr{O}\left\langle\mu, U_{1}, \ldots, U_{q}, 2^{-n}\right\rangle \mid U_{i} \in \mathscr{V}_{n}, \operatorname{dist}\left(U_{i}, U_{j}\right) \geq 3.2^{-n}\right.
$$

for $U_{i} \neq U_{j}$ and $\left.\min \left\{m_{i}, i=1, \ldots, q\right\} \geq(k+1)^{k} 2^{-n}\right\}$,

$$
\mathscr{U}_{n}=\bigcup_{i \geq n} \tilde{\mathscr{U}}_{i} \text { and } \mathscr{U}=\bigcup_{n=1}^{\infty} \mathscr{U}_{n} \text {. }
$$

Observe that $\mathscr{U}_{n}$ is an open cover of $P_{k}(X)$ for every $n \in \mathbf{N}$.

Note that for each simplex $\sigma \in \mathscr{N}(\mathscr{U})$ we have $\sigma=\left\langle V_{1}, \ldots, V_{p}\right\rangle$, where $V_{i}=\mathscr{O}\left\langle\mu_{i}, U_{1}^{i}, \ldots, U_{q(i)}^{i}, \varepsilon\right\rangle \in \mathscr{U}, \mu_{i}=\sum_{j=1}^{q(i)} m_{j}^{i} \delta x_{j}^{i}, x_{j}^{i} \in U_{j}^{i}, j=1, \ldots$, $q(i) ; i=1, \ldots, p$ and $\bigcap_{i=1}^{p} V_{i} \neq \varnothing$. Obviously we may assume that $V_{i} \in$ $\tilde{\mathscr{U}}_{n(i)}$ with $n(1) \leq n(2) \leq \cdots \leq n(p)$. Let us put $F_{i}=\left\{U_{1}^{i}, \ldots, U_{q(i)}^{i}\right\}$ for $i=1, \ldots, p$. Now define

$$
A(\sigma)=\left\{L=\left\{U^{i}\right\} \mid U^{i} \in F_{i}, \bigcap_{U^{i} \in L} U^{i} \neq \varnothing, \bigcap_{U^{i} \in L} U^{i} \cap U=\varnothing \text { for } U \notin L\right\} .
$$

(3.4) Lemma. Card $A(\sigma) \leq k$.

Proof. In fact we shall prove that $\operatorname{Card} A(\sigma)=q(p) \leq k$. From (9) it follows that every $U \in F_{p}$ belongs to at most one member $L \in A(\sigma)$. Therefore it suffices to establish that for every $L \in A(\sigma)$ there is a $U \in F_{p}$ such that $U \in L$. This follows from the following fact which is a crucial step in the proof of our result.

(3.5) Fact. If $m_{j(\bar{i})}^{\bar{i}} \geq(k+1)^{k} \varepsilon$, where $\varepsilon=\max \left\{\varepsilon_{i} \mid i=1, \ldots, p\right\}$, then for each $i \neq \bar{i}$ there exists $U_{j(i)}^{i}, j(i) \leq q(i)$, such that $\bigcap_{i=1}^{p} U_{j(i)}^{i} \neq \varnothing$.

Proof. Let $\mu \in \bigcap_{i=1}^{p} V_{i}$ with $\mu=\sum_{i=1}^{r} m_{i} \delta_{x_{i}}, r \leq k$. For simplicity we assume that $\bar{i}=1$. Since $\mu \in V_{1}$ it can be written in the form $\mu=\sum_{i=1}^{q(1)+1} \mu_{i}^{1}$, where $\mu_{i}^{1}=\mu \mid U_{i}^{1}, i=1, \ldots, q(1)$, and $\mu_{q(1)+1}^{1}=\mu \mid X \backslash \bigcup_{i=1}^{q(1)} U_{i}^{1}$. Observe that

$$
\left\|\mu_{q(1)+1}^{1}\right\|<2^{-n(1)} \leq \varepsilon \text { and }\|\mu\|=\sum_{i=1}^{q(1)+1}\left\|\mu_{i}^{1}\right\|=\sum_{i=1}^{r} m_{i}=1 .
$$

Write

$$
\mu_{1}=\sum_{i=1}^{q(1)} m_{i}^{1} \delta_{x_{i}^{1}}, \quad x_{i}^{1} \in U_{i}^{1}, \quad i=1, \ldots, q(1) .
$$

Since $\mu \in V_{1}$ we infer that ||$\left|\mu_{j(1)}^{1} \|-m_{j(1)}^{1}\right|<2^{-n(1)} \leq \varepsilon$. Therefore by (9)

$$
\left\|\mu_{j(1)}^{1}\right\| \geq m_{j(1)}^{1}-2^{-n(1)} \geq(k+1)^{k} \varepsilon-\varepsilon=\left((k+1)^{k}-1\right) \varepsilon .
$$

We write

$$
\mu_{j(1)}^{1}=\sum_{i=1}^{s} m_{i}^{1} \delta_{x_{i}^{1}}, \quad x_{i}^{1} \in U_{j(1)}^{1} \text { for } i=1, \ldots, s
$$


Then we have $\left\|\mu_{j(1)}^{1}\right\|=\sum_{i=1}^{s} m_{i}^{1}$. Put $A_{1}=\operatorname{supp} \mu_{j(1)}^{1}$.

(3.6) Claim. Let $V_{2}=\mathscr{O}\left\langle\mu_{2}, U_{1}^{2}, \ldots, U_{q(2)}^{2}, \varepsilon_{2}\right\rangle$. Then there exists $j(2) \leq q(2)$ such that $A_{1} \cap U_{j(2)}^{2} \neq \varnothing$.

Proof. Write $\mu=\sum_{i=1}^{q(2)+1}$, where $\mu_{i}^{2}=\mu \mid U_{i}^{2}, i=1, \ldots, q(2)$, and $\mu_{q(2)+1}^{2}=$

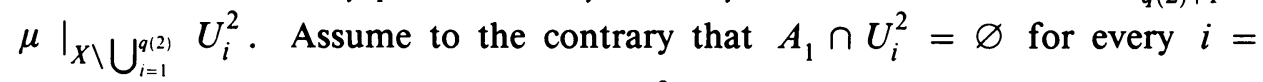
$1, \ldots, q(2)$. Then we have $A_{1} \subset \operatorname{supp} \mu_{q(2)+1}^{2}$.

Since $\mu \in V_{2}$, it follows that $\left\|\mu_{j(1)}^{1}\right\|=\left\|\mu\left|A_{1}\|\leq\| \mu\right| \operatorname{supp} \mu_{q(2)+1}^{2}\right\|<\varepsilon_{2} \leq \varepsilon$ which contradicts $(11)$, so the claim is proved.

Denote $I(2)=\left\{i \in\{1, \ldots, q(2)\} \mid U_{i}^{2} \cap A_{1} \neq \varnothing\right\}, B(2)=\bigcup_{i \in I(2)} U_{i}^{2}$, and $\widetilde{A_{2}}=A_{1} \cap B(2)$. Since $A_{1} \backslash \widetilde{A_{2}} \subset X \backslash \bigcup_{i=1}^{q(2)} U_{i}^{2}$ it follows that $\left\|\mu \mid A_{1} \backslash \widetilde{A_{2}}\right\| \leq \varepsilon_{2} \leq$ $\varepsilon$. Therefore from (11) we get

$$
\left\|\mu\left|\tilde{A}_{2}\|\geq\| \mu\right| A_{1}\right\|-\left\|\mu \mid A_{1} \backslash \tilde{A}_{2}\right\| \geq\left((k+1)^{k}-1\right) \varepsilon-\varepsilon \geq k(k+1)^{k-1} \varepsilon .
$$

Since $\operatorname{Card} I(2) \leq k$ there exists a $j(2) \in I(2)$ such that $\left\|\mu \mid \tilde{A} \cap U_{j(2)}^{2}\right\| \geq$ $(k+1)^{k-1} \varepsilon$. Let us put $A_{2}=\tilde{A}_{2} \cap U_{j(2)}^{2}$. Continuing this process we get a finite sequence $A_{1} \supset A_{2} \supset \cdots \supset A_{p}$. Since Card $A_{1} \leq k$ we infer that for any $p \in N$, the family $\left\{A_{1}, \ldots, A_{p}\right\}$ consists of at most $k$ different sets. Therefore we have $\left\|\mu \mid A_{p}\right\| \geq(k+1)^{k-(k-1)} \varepsilon=(k+1) \varepsilon>0$. In particular we get $\bigcap_{i=1}^{p} U_{j(1)}^{i} \supset A_{p} \neq \varnothing$.

This proves 3.5 .

Now we are already in a position to complete the proof of Theroem 3.1.

Assume that $K \prec\left\{\mathscr{U}_{n}\right\}$. Let $f: K^{0} \rightarrow P_{k}(X)$ be a selection. For each $V=$ $\mathscr{O}\left\langle\mu, U_{1}, \ldots, U_{q}, \varepsilon\right\rangle \in K^{0}$ we put $g_{0}(V)=\mu$. Assume that $\sigma=\left\langle V_{1}, \ldots, V_{p}\right\rangle \in$ $K$ with

$$
V_{i}=\mathscr{O}\left\langle u_{i}, U_{1}^{i}, \ldots, U_{q(1)}^{i}, \varepsilon_{i}\right\rangle \in \tilde{\mathscr{U}}_{n(i)}, \quad(\text { see }(9)),
$$

and $n(1) \leq n(2) \leq \cdots \leq n(p)$. Then $\bigcap_{i=1}^{p} V_{i} \neq \varnothing$. Observe that $\mu_{i}$ can be written in the form

$$
\mu_{i}=\sum_{U \in F_{i}} m(U) \delta_{x(U)}
$$

where $F_{i}=\left\{U_{1}^{i}, \ldots, U_{q(i)}^{i}\right\}_{i=1}^{p}$. As we have seen, for every $U^{p} \in F_{p}$ there exists a unique $L=L\left(U^{p}\right) \in A(\sigma)$ such that $U^{p} \in L$. For every $U \in F_{i}$ we put $J(U)=\left\{j \mid\left(U_{j}^{p}, U\right) \subset L\left(U_{j}^{p}\right)\right\}$. We shall define for each $i=1, \ldots, p$ a sequence $\left\{\hat{m}_{j}^{i}, \hat{x}_{j}^{i}\right\}_{j=1}^{q(p)}$ as follows: put

$$
\hat{x}_{j}^{i}= \begin{cases}x_{j}^{p}, & \text { if } L\left(U_{j}^{p}\right) \cap F_{i}=\varnothing, \\ x(U), & \text { if } U \in L\left(U_{j}^{p}\right) \cap F_{i} .\end{cases}
$$

Note that $\hat{x}_{j}^{i}$ is well defined, since $L\left(U^{p}\right) \cap F_{i}$ consists of at most one element for every $U^{p} \in F_{p}$ and for every $i=1, \ldots, p$. 
Now we put $\hat{m}_{j}^{i}=0$ if $L\left(U_{j}^{p}\right) \cap F_{i}=\varnothing$, and if $L\left(U_{j}^{p}\right) \cap F_{i} \neq \varnothing$ we define $\hat{m}_{j}^{i}$ by the following equalities:

$$
\sum_{j \in J(U)} m_{j}^{i}=\hat{m}(U), \quad \frac{\hat{m}_{j}^{i}}{m_{j}^{p}}=\frac{\hat{m}_{j^{\prime}}^{i}}{m_{j^{\prime}}^{p}} \text { for all } j, j^{\prime} \in J(U) .
$$

Then $\mu_{i}$ can be written in the form

$$
\mu_{i}=\sum_{j=1}^{q(p)} \hat{m}_{j}^{i} \delta_{\hat{x}_{j}^{i}} \quad \text { for } i=1, \ldots, p .
$$

We now define $g_{\sigma}: \sigma \rightarrow P_{k}(X)$ as follows: For each $x \in \sigma$ we have $x=$ $\sum_{i=1}^{p} \lambda_{i} V_{i}, \lambda_{i} \geq 0$ and $\sum_{i=1}^{p} \lambda_{i}=1$. We put

$$
g_{\sigma}(x)=\sum_{j=1}^{q(P)} m_{j} \delta_{x_{j}}
$$

where

$$
x_{j}=\sum_{i=1}^{p} \lambda_{i} \hat{x}_{j}^{i} \text { and } m_{j}=\sum_{i=1}^{p} \lambda_{i} \hat{m}_{j}^{i}
$$

for every $j=1, \ldots, q(P)$. Observe that by (7), $x_{j}$ is well defined. Now

$$
\begin{aligned}
\sum_{j=1}^{q(p)} m_{j} & =\sum_{j=1}^{q(p)} \sum_{i=1}^{p} \lambda_{i} \hat{m}_{j}^{i}=\sum_{i=1}^{p} \lambda_{i} \sum_{j=1}^{q(p)} \hat{m}_{j}^{i} \\
& =\sum_{i=1}^{p} \lambda_{i} \sum_{U \in F_{i}} m(U)=\sum_{i=1}^{p} \lambda_{i}=1 .
\end{aligned}
$$

Therefore $g_{\sigma}(x) \in P_{k}(X)$.

It is easy to see that for every $\sigma, \sigma^{\prime} \in K$ we have $g_{\sigma}\left|\sigma \cap \sigma^{\prime}=g_{\sigma^{\prime}}\right| \sigma \cap \sigma^{\prime}$ and $g_{\sigma} \mid \sigma^{0}=g_{0}$. Therefore the family $\left\{g_{\sigma}\right\}_{\sigma \in K}$ induces a map $g: K \rightarrow P_{k}(X)$ such that $g \mid K^{0}=g_{0}$.

We show that $\left\{\sigma_{n}\right\}$ satisfies Theorem 3.3. Assume that $\left\{\sigma_{n}\right\}$ is a sequence of simplices of $K$ such that $f\left(\sigma_{n}^{0}\right) \rightarrow \mu_{0} \in P_{k}(X)$ as $N\left(\sigma_{n}\right) \rightarrow \infty$.

Let $V=\mathscr{O}\left\langle\mu_{0}, W_{1}, \ldots, W_{q}, \varepsilon\right\rangle$ be a neighborhood of $\mu_{0}=\sum_{i=1}^{q} m_{i}^{0} \delta_{x_{i}^{0}}$, where $W_{i} \in \mathscr{W}$ for $i=1, \ldots, q$ are disjoint neighborhoods of $x_{i}^{0}, i=$ $1, \ldots, q$, respectively. Since $f\left(\sigma_{n}^{0}\right) \rightarrow \mu_{0}$ and $N\left(\sigma_{n}\right) \rightarrow \infty$, we infer that $g\left(\sigma_{n}^{0}\right) \rightarrow \mu_{0}$.

Note that by (7), (8), and the definition of $g$, there is an $n_{0} \in \mathbf{N}$ such that if $N\left(\sigma_{n}\right) \geq n_{0}$ and $x \in \sigma_{n}$, then we have

$$
g(x)=\sum_{i=1}^{q+1} \mu_{i}(x)
$$


where $\mu_{i}(x)=g(x)\left|W_{i}, i=1, \ldots, q, \mu_{q+1}=g(x)\right| X \backslash \bigcup_{i=1}^{q} W_{i}, \mid m_{i}^{0}-$ $\left\|\mu_{i}(x)\right\| \mid<\varepsilon$ for every $i=1, \ldots, q$, and $\left\|\mu(x)_{q+1}\right\|<\varepsilon$.

Therefore $g\left(\sigma_{n}\right) \rightarrow \mu_{0}$ as $N\left(\sigma_{n}\right) \rightarrow \infty$. Consequently by Theorem 3.3 we conclude that $P_{k}(X)$ is an ANR. This completes the proof of the theorem.

\section{REFERENCES}

[BP] C. Bessaga and A. Pelczynski, Selected topics in infinite dimensional topology, Warszawa 1975.

[DT] T. Dobrowolski and H. Torunczyk, Separable complete ANR's admitting a group structure are Hilbert manifolds, Topology Appl. 12 (1984), 229-235.

[Fe] V. V. Fedorchuk, Probability measures and absolute neighborhood retracts, Soviet Math. Dokl. 22 (1980), 849-853.

[Fr] A. H. Frink, Distance functions and the metrization problem, Bull. Amer. Math. Soc. 43 (1937), 133-142.

[M] H. W. Martin, A note on Frink metrization theorem, Rocky Mountain J. Math. 6 (1976), 155-157.

[N1] Nguyen To Nhu, Investigating the ANR-property of metric spaces, Fund. Math. 124 (1984), 243-254.

[N2] __ Hyperspaces of compact sets in metric linear spaces, Topology Appl. 22 (1986), 109-122.

[N3] _ Orbit spaces of finite groups acting linearly on normed spaces, Bull. Ac. Pol. Math. 32 (1986), 417-424.

[T] H. Torunczyk, Characterizing Hilbert space topology, Fund. Math. 111 (1981), 247-262.

(Current address of both authors): Institute of Mathematics, Vien Toan Hoc, P. O. Box 631, Bo Ho. HaNol, Vietnam 